\title{
High resolution terahertz spectroscopy for medical, biological and agricul- tural applications
}

\author{
$\underline{\text { V. L. Vaks }}^{1,2}$, E. G. Domracheva ${ }^{1}$, S. I. Pripolzin ${ }^{1}$, M. B. Chernyaeva ${ }^{1}$ \\ ${ }^{1}$ Institute for Physics of Microstructures RAS, Nizhny Novgorod, Russia, elena@ipmras.ru \\ ${ }^{2}$ Lobachevsky University, Nizhny Novgorod, Russia
}

High resolution terahertz spectroscopy [1] is promising method for analysis of multicomponent gas mixtures including biological ones. Among the spectroscopic methods, the only approach to date that ensures a near-theoretical-limit sensitivity along with a good spectral resolution limited just by the Doppler effect is the nonstationary spectroscopy based on free dumping polarization effect. Other advantages of the spectrometers include easy-to-use configuration and measurement time of several microseconds that provide registration of unstable gases. Application of this method will also benefits in registration of gasmarkers absorption lines at one shot without overlapping effect and performing minimal measuring time of few microseconds.

Exhaled breath analysis is a rapidly developing field of noninvasive medical diagnostics. To date, more than 800 substances have been identified among the products of physiological and biochemical processes in the human body. Their concentrations reflect the level of homeostasis, as well as the severity of pathologies and diseases [2,3]

The high-resolution $\mathrm{THz}$ spectroscopy method shows considerable promise for fast qualitative and quantitative analysis of various substances (metabolites) appeared in human organism including the breath at specific disease (in particular, cancer diseases, diabetes) for diagnostics at earlier stages or monitoring of patient's state. It allows to control efficiency of current treatment.

The application of high-resolution $\mathrm{THz}$ spectroscopy method for medicine diagnostics was used for monitoring the variation of the NO concentration at the radiotherapy course (the absence of nitric oxide in exhaled air of healthy people and its appearance in exhaled air of oncopatients with cancer of lung which had radiotherapy are determined at clinical tests); in the diagnostics and monitoring of the inflammations.

The method of detection of acetone in exhaled breath for earlier diabetes diagnostics shows its competitive ability in comparison with other methods. The experiments of detection of exhaled methanol, ethanol and $\mathrm{H}_{2} \mathrm{~S}$ and also experiments of simultaneous detection of acetone in exhaled breath and urine samples of diabetes patients show considerable promise for determination of metabolites-markers sets in exhaled breath and vapors of biological liquids unambiguously characterizing the diabetes presence. The results of simultaneous measurements of acetone concentrations at exhaled breath and urine samples of the diabetes patients at the frequency of $151646.597 \mathrm{MHz}$ are presented in Fig.1.

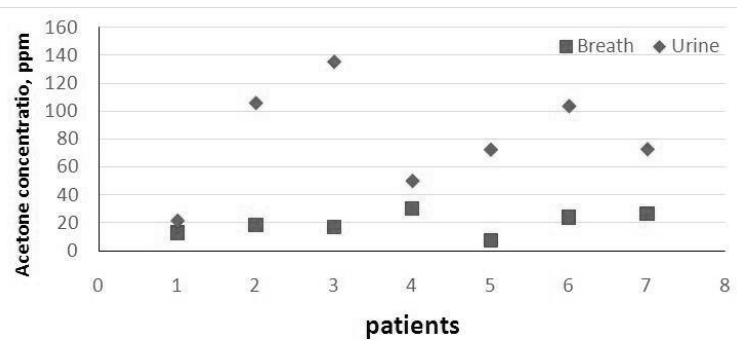

Fig. 1. Simultaneous measurements of acetone concentrations at exhaled breath and urine samples at the frequency of $151646.597 \mathrm{MHz}$

The possibility of use of terahertz high resolution nonstationary spectroscopy in such agricultural applications as veterinary diagnostics of diseases and pathology states of animals based on analysis of exhaled breath and vapors of biological liquids (urine, saliva); diagnostics of degree of corn mycosis based on analysis of corn odors; monitoring the quality of foodstuff (meat, fish, poultry) based on analysis of its odors shows considerable promise. The investigations of odors of meat and fish samples in fresh and natural decay with using the terahertz high resolution nonstationary spectroscopy methods for were carried out. The results reveal that odor of fresh meat contains ethyl formate, diethyl ether, alanine and glycine, that disappear during natural decomposition process. The latter is characterized by appearing the sulfur compound such as hydrogen sulfide and sulfur dioxide. After that the decaying process is going on with ammonia and nitrogenous organic substances appearance (formamide, ethylamine).In the spoiled fish odor propylene glycol, urethane, hydrogen sulfide, sulfur dioxide and nitriles substances have been found. The high resolution $\mathrm{THz}$ spectroscopy as "electronic nose" has been demonstrated to be very promising for food quality analyzing. [4]

\begin{tabular}{|l|l|}
\hline Grain & Substances \\
\hline $\begin{array}{l}\text { Winter wheat (Nemchi- } \\
\text { nivskaya-17) }\end{array}$ & $\begin{array}{l}\text { Glycolaldehyde } \\
\left(\mathrm{HCOCH}{ }_{2} \mathrm{OH}\right)\end{array}$ \\
\cline { 2 - 2 } & Formic acid $(\mathrm{HCOOH})$ \\
\hline Summer wheat (Sitara) & Formamide $\left(\mathrm{NH}_{2} \mathrm{CHO}\right)$ \\
\hline
\end{tabular}

The preliminary results on studying the grain odors composition of winter and summer wheats are presented in the Table. Some chemical substances, which can be results of defeating the grain by necrotrophic pathogens (formamide) or results of seed decomposition at heating, were identified. 
Functions of biological molecules (biomolecules) such as DNA and proteins, depend not only on their chemical composition, but also on the spatial structure of the molecules - the so-called configurational and conformational state. The study of configurational and conformational structure of biological molecules is an important task of biology, medicine. Monitoring the conformational transitions occurring during biologically important reactions, such as enzymatic catalysis, will allow a better understanding of the mechanisms of these reactions. Detection of biomolecules with pathological conformations in biological products (blood or cerebrospinal fluid samples) is important for early diagnosis of some diseases, such as prion infections., there are low-frequency molecular vibrations, in which large groups of atoms in a molecule move as a unit (e.g., nucleotide in DNA) in the $\mathrm{THz}$ frequency region The frequency of these oscillations depends on the structure of the whole molecule. Oscillation frequencies of hydrogen bonds supporting the structure of biomolecules also lie in the $\mathrm{THz}$ frequency range. Therefore, $\mathrm{THz}$ absorption spectra contain specific information about the structure and properties of hydrogen bonds. Note that circular dichroism spectra allow one to decipher the structure of molecules and to reconstruct relative positions of atomic groups, but contain no information about hydrogen bonds. The water absorption lines also lie in the $\mathrm{THz}$ region. These are dependent on vibrational-rotational transitions and relaxational dielectric losses. Water absorption bands allow to estimate the amount of water in the sample.

Applying methods of $\mathrm{THz}$ absorption spectroscopy is difficult due to high complexity of spectra interpretation. There are no detailed spectroscopic databases for polymer molecules in the $\mathrm{THz}$ region to date. It is impossible to calculate polymer absorption spectra, taking into account every electron and every nucleus in the molecule. The measurements of $\mathrm{THz}$ spectra of DNA and modeling polymers were carried out with using the high resolution $\mathrm{THz}$ spectroscopy method [5].

The preliminary investigations of gas-markers in $\mathrm{THz}$ frequency range have demonstrated the advantages of the high resolution $\mathrm{THz}$ spectroscopy methods for various applications. This approach could be promising to become the compact high precise analytical tool.

This work was carried out in the framework of state targets N 0035-2014-0206 and financially supported by the Russian Foundation for Basic Research (grant N 18-42-520050 r_a_povoljie, N 17-00-00184 KOMFI, N18-52-16017)

\section{References}

1. V. Vaks, High-Precise Spectrometry of the Terahertz Frequency Range: The Methods, Approaches and Applications// Journal of Infrared, Millimeter and Terahertz Waves. 2012, V. 33, N. 1, P. 43-53..

2. Stepanov $E V$ Diodnaya Lazernaya Spektroskopiya i Analiz Molekul-Biomarkerov (Diode Laser Spectroscopy and Analysis of Biomarker Molecules) (Moscow: Fizmatlit, 2009)

3. VL Vaks, E G Domracheva, E A Sobakinskaya, $M B$ Chernyaeva. Exhaled breath analysis: physical methods, instruments, and medical diagnostics //Physics-Uspekhi 2014, V. 57, N 7, P. $684-701$

4._V.L.Vaks, M.B.Chernyaeva, E.G.Domracheva and S.I.Pripolzin. Multifrequency laser spectroscopy of biological tissues odors// Proceedings of V International Symposium TOPICAL PROBLEMS OF BIOPHOTONICS, 20 24 July, 2015, Nizhny Novgorod, Russia pp.218-219

5. Vaks, V.L., Semenova, A.V., Guseva, Y.S., Panin, A.N. Phenomenological model and experimental study of DNA absorption spectra in THz range //Opt. Quant. Electron. 2017, V. 49, N 5, P. 193 (1-24). 